\title{
Gender matters! Analyzing global cultural gender preferences for venues using social sensing
}

Willi Mueller ${ }^{1 \dagger}$, Thiago H Silva ${ }^{2,3^{*}+}$, Jussara M Almeida ${ }^{2}$ and Antonio AF Loureiro ${ }^{2}$

\author{
*Correspondence: \\ thiagohs@dcc.ufmg.br \\ ${ }^{2}$ Department of Computer Science, \\ Universidade Federal de Minas \\ Gerais, Belo Horizonte, MG, Brazil \\ ${ }^{3}$ Present address: Department of \\ Informatics, Universidade \\ Tecnológica Federal do Paraná, \\ Curitiba, PR, Brazil \\ Full list of author information is \\ available at the end of the article \\ ${ }^{\dagger}$ Equal contributors
}

\begin{abstract}
Gender differences is a phenomenon around the world actively researched by social scientists. Traditionally, the data used to support such studies is manually obtained, often through surveys with volunteers. However, due to their inherent high costs because of manual steps, such traditional methods do not quickly scale to large-size studies. We here investigate a particular aspect of gender differences: preferences for venues. To that end, we explore the use of check-in data collected from Foursquare to estimate cultural gender preferences for venues in the physical world. For that, we first demonstrate that by analyzing the check-in data in various regions of the world we can find significant differences in preferences for specific venues between gender groups. Some of these significant differences reflect well-known cultural patterns. Moreover, we also gathered evidence that our methodology offers useful information about gender preference for venues in a given region in the real world. This suggests that gender and venue preferences observed may not be independent. Our results indicate that our proposed methodology could be a promising tool to support studies on gender preferences for venues at different spatial granularities around the world, being faster and cheaper than traditional methods, besides quickly capturing changes in the real world.
\end{abstract}

Keywords: gender preferences for venues; social media; large-scale assessment

\section{Introduction}

Gender differences can be considered one of the great puzzles of modern society. It has a subjective nature, and may vary greatly across cultures [1-3]. For instance, when comparing different regions of the world, women and men often differ in their assumed capacities, and others. This makes gender differences hard to explain. Indeed, over the past decades, this topic has received a lot of attention in the area of Social Science, but there is still a long way to a consensus on the subject $[4,5]$.

In order to study the differences between gender groups around the world, social scientists often rely on manual methods to gather heterogeneous data, often using surveys with volunteers. The collected data may then be aggregated to compute particular metrics, such as the Gender Inequality Index (GII) developed by the United Nations Development Programme (UNDP) [6].

(c) The Author(s) 2017. This article is distributed under the terms of the Creative Commons Attribution 4.0 International License (http://creativecommons.org/licenses/by/4.0/), which permits unrestricted use, distribution, and reproduction in any medium, provided you give appropriate credit to the original author(s) and the source, provide a link to the Creative Commons license, and indicate if changes were made. 
However, these traditional methods are time-consuming because of the manual steps. Moreover, data produced under such conditions are commonly released after long time intervals (e.g., it could take several years). Therefore, they cannot quickly capture changes in the dynamics of societies. Besides, the results from cross-regional gender differences studies, such as the GII reports, are usually available only for large geographic regions, often countries. Thus, even though survey-based studies could be carried out in arbitrary small regions, such as a city, a neighborhood or even a particular venue (e.g., a university or a mall), information about gender differences at such fine spatial granularities is not easily available.

With that, one of the main research questions of this paper is: Can we propose a complementary method to help in the study of gender differences in a large scale and in a faster way than traditional methods?

Location-based social networks (LBSNs), such as Foursquare, ${ }^{\mathrm{a}}$ are currently very popular, mostly due to the widespread use of smartphones around the world. In such applications, users implicitly express their preferences for locations by performing check-ins at specific venues. Check-ins can then be seen as a source of social sensing, capturing how people behave in the real world with respect to the places they often visit. As discussed in $[7,8]$, such signals can be explored to better understand human dynamics in urban areas, and, particularly, culture-related urban patterns.

We focus on a particular aspect of the culture of a society, namely gender bias [2, 3, 914]. We aim at investigating whether user check-ins in LBSNs can also be used to assess cultural gender preferences for venues at different urban regions of the physical world. In our context, culture is expressed through preference for a particular venue. To capture that, we propose a methodology to quantify the differences between male and female users in preferences for particular venues. The aggregation of such differences over multiple venues could then be used, for example, in the construction of an indicator of gender differences in a given region.

We illustrate the use of our methodology by extracting user preferences for venues located in different urban regions around the world from check-in data collected from Foursquare. We then identify significant differences for specific venues between gender groups in various regions, which suggest that gender and venue preferences may not be independent in those regions. We illustrate the potential use of our methodology by applying it to various spatial granularities, including countries, cities, and a particular type of venues in a given city.

We demonstrate one application that aims at identifying groups of similar urban areas according to the degree of gender preference for venues observed in different (types of) places located in those areas. Furthermore, we investigate to which extent gender preferences for venues are related to gender differences. For that, we compared our results with those produced using the United Nations GII values. This analysis suggests that our approach might capture some essential aspects of gender differences. Besides, it also motivates the study of new approaches to using social sensing jointly with other data in future developments of gender differences indices.

In summary, the main contributions of this work are: (i) a methodology to characterize gender preferences for venues in different regions at different spatial granularities, around the world, based on LBSNs and (ii) a study of our methodology as a means to assess cultural gender preferences for venues showing its potential for different studies in several areas. 
The results that our methodology produces could be a promising tool to support largescale gender preferences for venues studies that require less human effort and time, compared with traditional methods, and can quickly react to changes in the real world because it relies on LBSNs data. The obtained results could be used in several contexts. For instance, they might help policy makers to evaluate the effect of implemented policies regarding the minimization of gender differences in certain regions/venues of the city. Similarly, they might help business owners and marketers to better understand their consumers. For example, if a coffee shop has a very distinct pattern of consumer gender compared with other coffee shops in the same city, the owner could exploit this knowledge to promote advertisement. Our method may also be used to identify similarities and discrepancies regarding venue preferences of gender groups across different regions. Finally, the results might drive the design of more culturally-aware venue recommender systems, as men and women may have different preferences in regions with distinct cultures.

The rest of this article is organized as follows. Section 2 review the related work. Section 3 introduces our dataset, while Section 4 presents a study about gender preferences for venues in urban regions of different sizes. Section 5 presents some applications that could benefit from our work. Section 6 compares our results with official indices of gender differences. Section 7 discusses some of the known limitations of our study. Finally, Section 8 presents the concluding remarks and future work.

\section{Related work}

The study of gender differences has been receiving a considerable amount of attention in different areas. Some recent studies include the investigation of gender differences in education [15], in relationships [2,16], and with respect to the use of technology [17]. In the latter, the authors analyzed how 270 adults used the Web, aiming at identifying differences in online activity. These prior studies, as most social science studies, relied on surveys with a reasonably small sample size. However, such manual approach imposes big challenges to studies with larger sample sizes (e.g., thousands or millions of users).

Recently, scientists are jointly applying techniques from Computer Science and Statistics to support sociological studies using large-scale datasets. For example, Kershaw et al. [18] looked into the use of social media to monitor the rate of alcohol consumption. Weber et al. [19] used web search query logs to analyze and visualize political issues. Some other topics of study include the understanding of city dynamics $[8,20,21]$, event detection/study [2227], cultural differences [7, 10, 28-30], and gender inference [31-33].

On the particular topic of cross-gender differences, Ottoni et al. [34] observed a great difference between female and male users with respect to their motivations for using Pinterest. Lou et al. [35] investigated how gender swapping is revealed in massively multiplayer online games, observing that both male and female players achieve higher levels in the game faster with a male avatar than with a female avatar. De Las Casas et al. [36] characterized the use of Google+ by members who declared themselves as neither female nor male individuals, but as other. Cunha et al. [37] studied gender distinctions in the usage of Twitter hashtags, concluding that gender can be considered a social factor that influences the user's choice of particular hashtags about a given topic. Garcia et al. [10] measured gender biases of dialogues in movies and social media, showing that Twitter presents a male bias, whereas MySpace does not. Wagner et al. [13] present a method for assessing gender bias on Wikipedia. Gender bias in Wikipedia is also studied by Graells-Garrido et 
al. [38]. Magno and Weber [12] study gender inequality through user participation in two online social networks, Twitter and Google+, finding, for example, that the gap between the number of users correlates with the gender gap index, i.e., countries with more men than women online are countries with higher gender difference. Volkovich et al. [14] also study gender difference in a large online social network, looking mainly in the way how men and women sign up to a social network platform and make friends online. They found a general tendency towards gender homophily, more marked for women.

In this work, we also use a large-scale dataset, in our case data from a popular LBSN, which expresses user preferences for venues in a region, for various regions around the globe. However, unlike the aforementioned prior studies, we want to infer relevant crossgender differences in the physical world, instead of online. To that end, we propose a methodology to quantify the differences between male and female users in preferences for particular venues across different cultures.

\section{Dataset description}

A common approach to conducting studies on human behavior is by means of surveys, where participants answer questions administered through interviews or questionnaires [39-41]. However, despite its wide adoption, survey-based studies do have some severe constraints, which are well known to researchers. First, they may be costly and do not scale up. It is often hard to obtain data of millions or even thousands of people, particularly when focusing on multiple geographic regions. Second, they provide static information, reflecting human behavior at a specific point in time. Thus, they cannot capture well the natural changes we may expect from dynamic societies.

Instead of relying on survey data, we here investigate the use of publicly available data from LBSNs, notably Foursquare, to study gender preference for venues. LBSNs can be accessed everywhere by anyone with an Internet connection, solving the scalability problem and allowing the collection of data from (potentially) the entire world [21]. Moreover, these systems are quite dynamic, capturing behavioral changes of their users when they occur.

Nevertheless, the use of LBSN data also has some limitations, such as an inherent bias to regions and population groups where the application and required technology are more widely used. Yet, recent work has exploited this type of data to support social studies on various topics, as further discussed in Section 2. We here focus on gender, and investigate its use to drive studies on gender preferences for venues.

Specifically, our dataset consists of check-ins made by Foursquare users and become publicly available through Twitter between April 25th and May 1st 2014. This dataset contains roughly 2.9 million tweets with check-ins shared by approximately 630 thousands users. Foursquare venues are grouped into ten categories (in parenthesis are the abbreviations used here): Arts \& Entertainment (Arts); College \& University (Education); Event; Food; Nightlife Spot (Nightlife); Outdoors \& Recreation; Professional \& Other Places (Work); Residence; Shop \& Service; Travel \& Transport. Each category, in turn, has several subcategories. For example, Comedy Club, Museum, and Casino are subcategories of Arts. Bar, Rock Club, and Pub are subcategories of Nightlife. College Lab, Fraternity House, and Student Center are subcategories of Education. Finally, Baseball Stadium, Surf Spot, and Park are subcategories of Outdoors \& Recreation.

We applied the following filters to our dataset: We only considered check-ins performed by users who specified either "male" or "female" as gender in their Foursquare profiles. We 
Table 1 Overview of our dataset

\begin{tabular}{lrrr}
\hline Country & Check-ins (\% By male users) & Venues & Users (\% male) \\
\hline Brazil & $29,017(49 \%)$ & 3,042 & $20,164(49 \%$ male) \\
France & $422(60 \%)$ & 38 & $337(61 \%$ male) \\
Germany & $329(76 \%)$ & 35 & $309(77 \%$ male) \\
Japan & $12,326(86 \%)$ & 1,028 & $7,919(85 \%$ male) \\
Kuwait & $2,816(45 \%)$ & 243 & $2,308(45 \%$ male) \\
Malaysia & $29,599(56 \%)$ & 2,685 & $17,101(54 \%$ male) \\
Mexico & $29,963(59 \%)$ & 2,892 & $19,660(59 \%$ male) \\
Saudi Arabia & $3,576(39 \%)$ & 342 & $2,714(39 \%$ male) \\
South Korea & $297(39 \%)$ & 33 & $250(42 \%$ male) \\
Spain & $467(74 \%)$ & 58 & $432(74 \%$ male) \\
Thailand & $14,579(23 \%)$ & 1,346 & $8,772(23 \%$ male) \\
United Arab Emirates & $211(55 \%)$ & 27 & $187(56 \%$ male) \\
United Kingdom & $1,061(69 \%)$ & 115 & $920(70 \%$ male) \\
United States & $15,633(60 \%)$ & 1,756 & $11,686(61 \%$ male) \\
Turkey & $29,369(54 \%)$ & 1,470 & $26,336(53 \%$ male) \\
\hline
\end{tabular}

disregarded all check-ins in venues with fewer than five check-ins and considered only one check-in per user per venue to avoid users with many check-ins biasing the popularity of a venue among all users. Moreover, we considered only venues in the Arts, Education, Food, Nightlife, and Work categories, which we expect to better capture differences in gender preferences for venues in a society. We discarded categories that have many subcategories with expected biases towards a particular gender (e.g., Men's Store) as well as categories covering places that might be more popular among non-locals (e.g., hotels and airports), as our goal is to identify gender patterns among residents of particular regions.

Furthermore, when analyzing a particular region, we only considered venues of a given subcategory if there are at least two different venues of that subcategory meeting the aforementioned filter criteria in the given region. Finally, we selected 15 countries covering different regions of the world: Brazil, Mexico, and United States (America); France, Germany, Spain, and United Kingdom (Europe); Japan, Malaysia, South Korea, and Thailand (East and South Asia); Kuwait, Saudi Arabia, Turkey, and United Emirates Arab (Western and Middle-East Asia). To ease the computational effort we kept the number of check-ins per country below 30,000 by randomly sampling check-ins belonging to a fixed number of venues. This step was only necessary for Turkey and Malaysia.

The filtered dataset, which is used in our analyses, contains a total of 170,665 check-ins performed by 118,902 users in 14,982 venues, distributed across 15 countries, as detailed in Table 1. We note that male users account for at least half of all check-ins in 10 of the selected countries. The number of subcategories that passed in our filtering criteria for each country are: 126 for Brazil; 9 for France; 12 for Germany; 74 for Japan; 34 for Kuwait; 116 for Malaysia; 129 for Mexico; 38 for Saudi Arabia; 11 for South Korea; 15 for Spain; 85 for Thailand; 95 for Turkey; 8 for the United Arab Emirates; 28 for the United Kingdom; and 120 for the United States.

\section{Characterization of cultural gender preferences for venues}

In this section, we present our methodology to analyze gender preferences for venues in different regions around the world, which are known to present some cultural differences [42]. We start by introducing our methodology (Section 4.1), and then illustrate how it is applied to study gender preferences for venues at the country level (Section 4.2) and at finer granularities (Section 4.3). 


\subsection{Proposed methodology}

\subsubsection{Estimating gender preferences}

The first step in our methodology is to characterize the preferences within each gender group for different locations in a given region. To that end, we extract check-ins in venues located in the region under study from Foursquare and use them to map the preferences of each gender for specific venues in the region. Our methodology is general enough to consider all venues of the same type (same subcategory) jointly, or each venue individually, depending on the goal of the study. In the following description, we consider the former, but in Section 4.3 we show how it can be easily applied to study cross-gender differences in preferences for individual venues.

Given each venue subcategory that passed our filtering criteria in the region under study (Section 3), we measure the popularity of all venues of that subcategory within each gender group. That is, given a region, a subcategory, a venue, and a gender, we compute the percentage of all check-ins by users of that gender in all venues of that region that were performed in venues of the given subcategory. To make the graphs better comparable, we normalize these percentages by dividing by the maximum value, only to ease the visualization.

The next step consists in computing the cross-gender popularity difference $d_{s}$ for each subcategory. Let us define a 2-dimensional space based on the two popularity measures (one per gender). The diagonal of this space represents an ideal case where popularity is balanced across genders. The cross-gender popularity difference for a given subcategory is then defined as the shortest Euclidean distance between the point representing that particular subcategory in the 2-dimensional space and its diagonal. ${ }^{\mathrm{b}}$ Differences below zero indicate greater popularity among female users as the point lies on the left side of the diagonal. In contrast, differences above zero imply greater popularity among male users.

Given a non-zero cross-gender popularity difference, computed as described, a natural question that emerges is: Is this difference related to a possible difference in size of the female/male population in the studied dataset, or does it reflect a significant gender-related pattern?

\subsubsection{Testing statistical significance}

To tackle this question, we built a null model using the following process: We count the number $c$ of all check-ins located in the region under study. Furthermore, we group all unique users in $U$ and all locations in $L$ (preserving the venue's attributes, i.e., subcategory, latitude, and longitude). After that, we generate $c$ check-ins randomly choosing for each of them a gender (female or male), a location in $L$, and a user in $U$. Any element (gender, location, or user) is randomly sampled with replacement and thus can be chosen more than once. In this way, we disjoint the correlation between the user, gender, and location. We then recompute the cross-gender popularity difference for each subcategory as discussed in Section 4.1.1.

We repeat this process $k=100$ times, producing a distribution of popularity differences for each subcategory. By comparing the observed difference for a given subcategory against the corresponding distribution produced by the aforementioned randomization process, we are able to rule out any possible effect due to differences in gender population sizes. Also, we can test whether the observed cross-gender difference is significant, meaning that it is indeed related to gender preferences. 


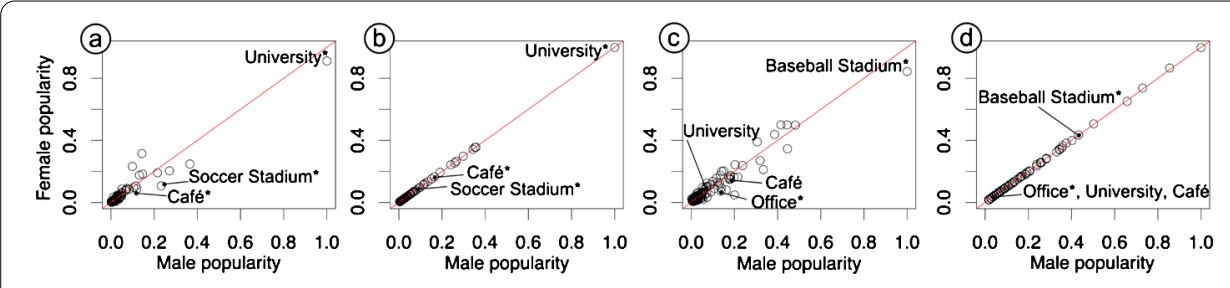

Figure 1 Popularity of subcategories within each gender. Popularity (normalized) of venue subcategories within each gender for (a) Brazil and (b) the USA as well as the average values after a null model creation for (c) Brazil and (d) the USA.

Let $d_{s}$ be the observed difference for subcategory $s$, and $D_{s}^{\text {null }}$ the distribution of differences obtained after randomization. We compare $d_{s}$ against $D_{s}^{\text {null }}$ with the minimum min and maximum max limits representing the values observed in $D_{s}^{\text {null }}$ with $99 \%$ of confidence. The observed difference is significant if it lies outside the range [min, max]. We refer to the range of values against which $d_{s}$ is tested as the acceptance range $\left[\Delta_{\min }, \Delta_{\max }\right]$. If $d_{s}$ lies inside this range, it cannot be considered significant, and we cannot tell whether it actually reflects a gender-related pattern.

We also tried another randomization approach, preserving all check-in attributes unchanged, except gender, and randomly shuffling $k=100$ times the gender associated with all check-ins located in the region under study. Yet, the results are similar to the discussed above. For this reason, in this study, we only present more details and discuss results of the approach mentioned previously. Next, we illustrate the use of our methodology in various scenarios.

\subsection{Country-level analysis}

We start by focusing on a coarser spatial granularity and use our methodology to analyze gender preferences for venue subcategories across different countries. Figure $1^{\mathrm{c}}$ shows the (normalized) popularity, within male and female users, of considered subcategories in Brazil (Figure 1(a)) and United States (Figure 1(c)). Each point in each graph represents a subcategory, which only some examples are labeled to avoid visual pollution. In Figure 1(a) and (c) soccer and baseball stadiums are the most popular subcategories, respectively, both biased towards male users.

We analyzed all subcategories that passed our filtering criteria in each country, but we here discuss only some of the most popular examples in terms of the number of check-ins: Baseball Stadium, Café, Cricket Ground, Office, Soccer Stadium, and University. Figure 2 shows the popularity difference of venue subcategories within each gender in all studied countries. To ease the comparison, the differences represent normalized values (into the range $[0,1])$ for each country. Note, that differences below zero indicate greater popularity among female users, while differences above zero indicate greater popularity among male users.

Studying the results in Figure 2, we can see, for instance, that Soccer Stadiums, tend to be more popular among male users in all countries except in Turkey. In contrast, Universities are more popular among male users in Brazil, but more female-oriented in Saudi Arabia. Similarly, there is a cross-gender difference towards men for Cafes in Turkey and the USA, whereas, in Malaysia and Saudi Arabia, those places tend to attract more female users. Do these differences reflect different gender preferences in those countries? 


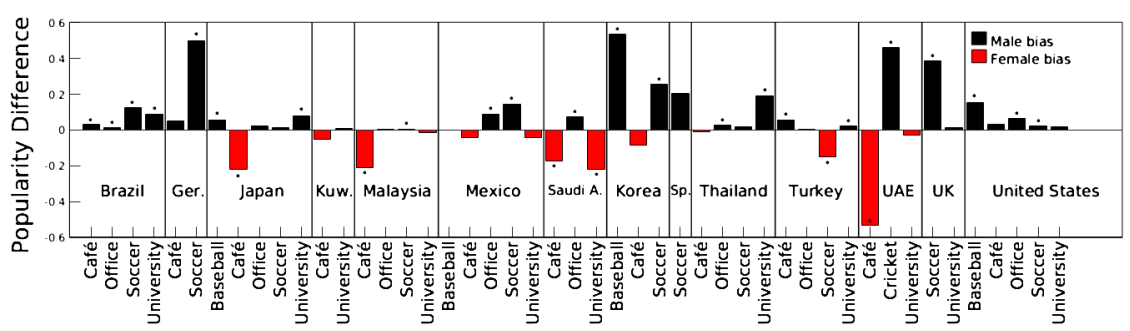

Figure 2 Popularity differences between genders for various countries. Popularity difference of venue subcategories within each gender in various countries. For each country we show the subcategories Baseball Stadium, Café, Cricket Ground, Office, Soccer Stadium, and University. The differences represent normalized values for each country, to facilitate the comparison.

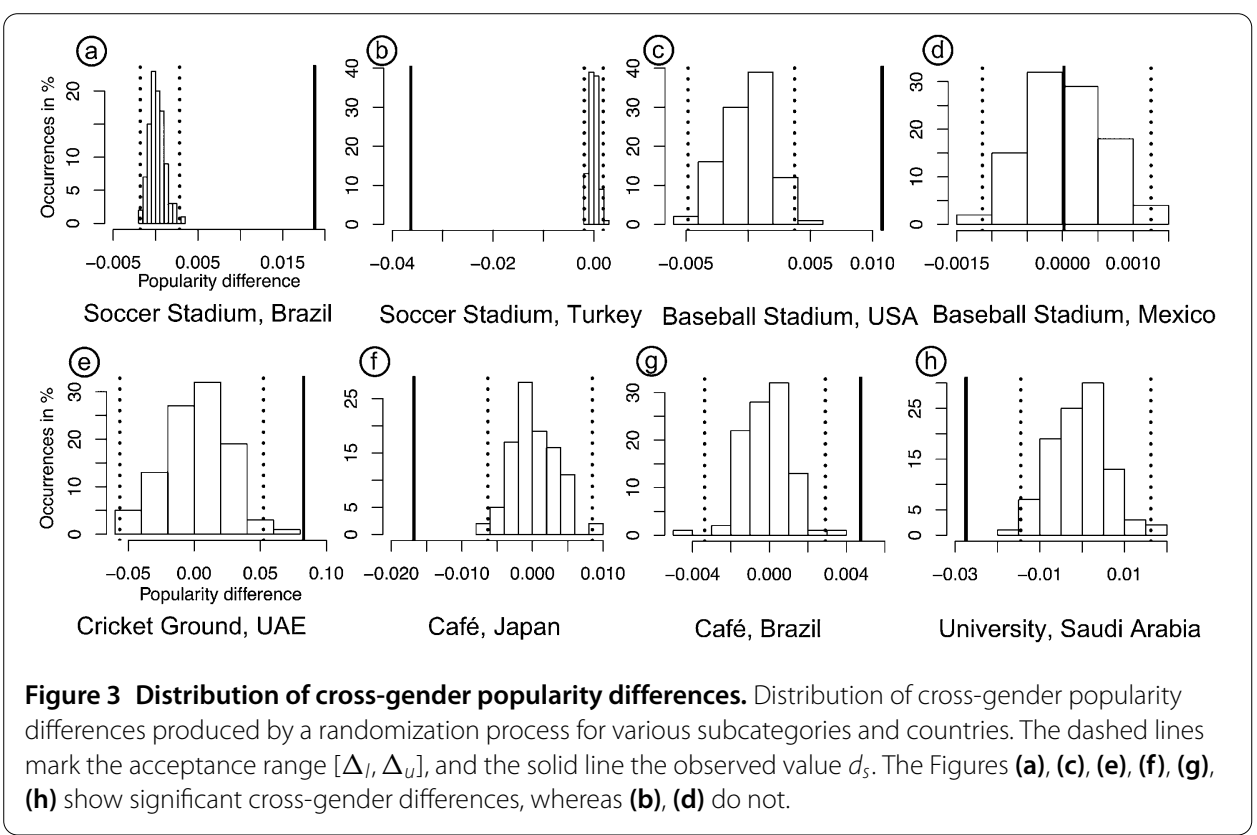

We then turn to the results produced after the randomization process, shown in Figure $1(\mathrm{~b})$ and (d), which presents average popularity values computed across all $k=100$ repetitions. Note that, unlike in the observed data, those values are well balanced across genders in all cases. This pattern repeats for all studied regions, for this reason, we only show two illustrative examples.

We delve further into some of the results shown in Figure 1, starting with three particular subcategories related to sports, namely Soccer Stadium, Baseball Stadium, and Cricket Ground. Out of all analyzed countries, we find that Soccer Stadiums are significantly more popular among male users, i.e. have statistically significant cross-gender differences above zero in Brazil, Mexico, Germany, South Korea, the USA, Malaysia and the UK. As an example, Figure 3(a) shows the distribution of the cross-gender differences computed during the randomization procedure for Brazil. The solid vertical line is the difference observed in the data $\left(d_{s}\right)$, whereas the dashed vertical lines indicate the acceptance range $\left[\Delta_{\min }, \Delta_{\max }\right]$. Note that the observed difference $(0.0188)$ by far exceed the upper limit $\Delta_{\max }$.

In contrast, in Spain, Japan, and Thailand, the cross-gender popularity differences were not significant, according to our test. This might be due to a greater popularity of the fe- 
male soccer teams in these countries, which attract proportionally more male users to related venues, compared to Brazil, Mexico and the other aforementioned countries. Turkey, however, is an interesting case: We found a difference significantly below zero, indicating a far higher preference among female users, result shown in Figure 3(b). This is most likely a consequence of a penalty, introduced in 2011, for Turkish soccer clubs that only women and children under 12 years are allowed to attend games of clubs sanctioned because unruly fans. ${ }^{\mathrm{d}}$ In fact, $90 \%$ of the 2,536 check-ins performed in Turkish soccer stadiums in our dataset were performed in the stadium of Fenerbace Istanbul. This club was affected by that penalty, being obligated to ban male teenagers and adults of its stadium during our collection period. During this period this club hosted a game over 50,000 spectators. ${ }^{\mathrm{e}}$

Turning our attention to the Baseball Stadium subcategory, we find that those venues are significantly more popular among male users in Japan, South Korea and the USA. The distribution of the cross-gender differences computed during the randomization procedure for this subcategory for the USA is shown in Figure 3(c). In contrast, in Mexico, we find no significant trend towards any gender, as shown in Figure 3(d).

The Cricket Ground subcategory was only analyzed for the United Arab Emirates (UAE), as venues in this subcategory in the other countries did not pass our filtering criteria. For that country, where this subcategory was the most popular type of sports-related venue, we did find a statistically significant positive cross-gender difference, indicating a greater popularity among male users (Figure 3(e)). Interestingly, a general result for all three sports subcategories is that the overall most popular subcategory of sports venues in the country is often significantly more male-oriented.

Regarding other venue subcategories, we find that Offices are significantly more popular among male users in all countries with sufficient data about this subcategory, but Turkey, Japan, and Malaysia. In the case of Malaysia, the exception might be due to the fact that most popular venues classified as Office are also located in shopping malls, which traditionally attract many women, thus compensating for any possible male bias. This also happens in Japan, and besides that, among the most popular offices there is a Korean-pop record label, a style that has a mostly female audience, ${ }^{\mathrm{f}}$ indicating that this office may attract many female fans.

Cafes, in turn, only have a significant cross-gender popularity difference in 6 out of 9 analyzed countries with sufficient data about cafes. While these places are female-oriented in Japan, Malaysia, Saudi Arabia, and the United Arab Emirates, they are more popular among male users in Brazil and Turkey. One possible reason that helps to explain this result is that most popular Cafes analyzed in Brazil are located in popular areas among men, such as offices and financial regions. In Turkey, it is usually men who most frequent cafes, although these also now welcome women [43]. We illustrate this finding by presenting the results for Japan and Brazil in Figure 3(f) and (g), respectively. These results illustrate significantly different cross-gender patterns in both countries.

As a final example, the subcategory University is significantly more popular among male users in Brazil, Japan, Thailand, and Turkey. Yet, in Saudia-Arabia University is significantly more female-oriented, as shown in Figure 3(h). One possible explanation for the latter is that the majority of university graduates are women in Saudi Arabia, according to a recent report. ${ }^{\mathrm{g}}$

Our goal in this section was to illustrate the use of the proposed methodology to characterize gender preferences for different types of locations in a country. As discussed above, 

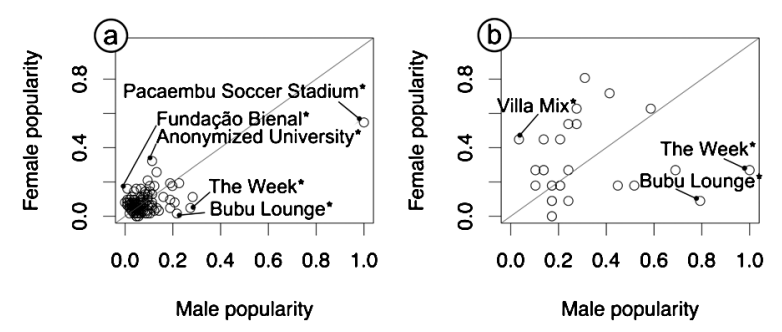

Figure 4 Popularity of individual venues within each gender in São Paulo, Brazil. Popularity (normalized) of individual venues within each gender group in São Paulo, Brazil: (a) all venues from all subcategories, (b) only venues from the subcategory Nightclub.

our results do suggest that the observed differences reflect inherent cultural aspects of each country.

\subsection{Finer grained analyses}

In the previous section, we showed how our methodology can be used to identify significant cross-gender differences in preferences for venues in different countries. We now show that it can also help identify such differences at much finer granularities. Focusing on a specific city - São Paulo (Brazil) - we study differences in gender preferences for specific venues in two scenarios: all venues in the city, and all venues of a given subcategory. The latter is useful to identify places where gender preferences patterns diverge from those of the same type in the city.

In the first scenario, we applied our methodology considering 2,422 check-ins at venues located in São Paulo. Figure 4(a) shows these results for the observed data (normalized just to ease the visual evaluation). As Figure 4(a) shows, there are some large cross-gender differences in the city. Out of all 248 venues analyzed, we identified 21 where the crossgender popularity difference is statistically significant, according to our methodology.

One such example is a private university, that explicitly requested to be anonymized. It is more popular among female users, with a statistically significant cross-gender difference below zero (Figure 5(a)). This might be explained by an often larger presence of women in the particular courses located on that campus (namely health, arts, pedagogy, and media production) in Brazil. Similarly, the Technology and Communications University FAPCOM, which offers similar and related courses, is also significantly more popular among female users. A spokesperson for the anonymized university confirmed via email that they indeed have $68 \%$ female students enrolled at the campus our method detected as anomalous.

Another example is the Art Museum Fundação Bienal Ibirapuera, which is also significantly more popular among female users, as shown in Figure 5(b). This result was confirmed by a spokesperson for this museum. Besides that, the result is consistent with findings from a recent survey performed with visitors of this museum, confirming that the majority of the public is female [44].

In the second scenario, we considered check-ins at individual Nightclub venues located in São Paulo. To ease the visualization of the results, they were plotted normalized. As shown in Figure 4(b), various nightclubs lie far from the diagonal. Yet, out of all 29 nightclubs analyzed, we found 4 with statistically significant cross-gender differences: The Week, BubuLounge, Villa Mix, and Blitz Haus. 

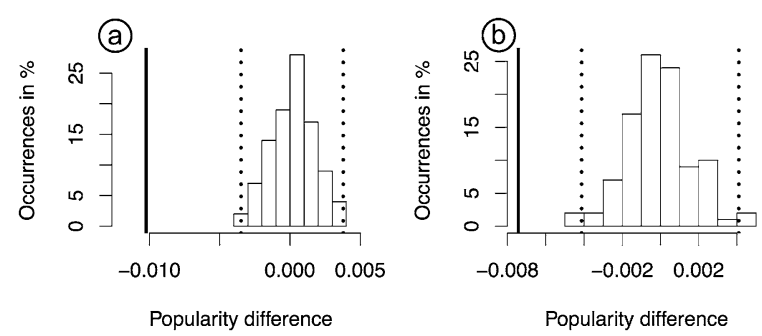

Figure 5 Randomized cross-gender popularity differences distribution for a university and an art museum. Distribution of cross-gender popularity differences produced by a randomization process for (a) an anonymized university campus and (b) the art museum Fundação Ibirapuera in São Paulo city.
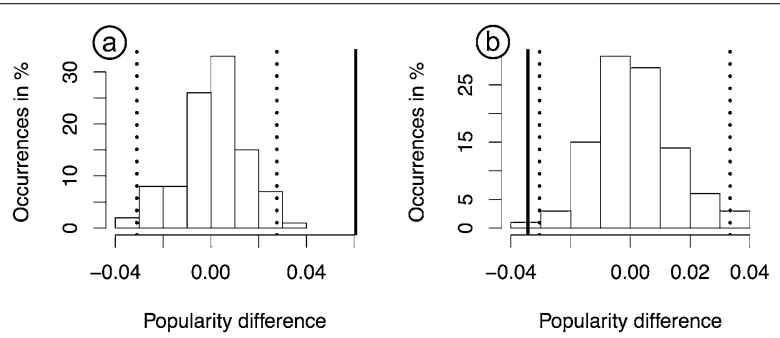

Figure 6 Randomized cross-gender popularity differences distribution for two nightclubs. Distribution of cross-gender popularity differences produced by a randomization process for the Nightclub venues (a) The Week and (b) Villa Mix in São Paulo city.

The Week (Figure 6(a)), and Bubu Lounge are significantly more male-oriented. Supporting our finding, today The Week and Bubu Lounge are classified as a Gay Bar on Foursquare, which was not the case during our data collection. Also, on similar recommendation platforms, such as Yelp, ${ }^{\mathrm{h}}$ TripAdvisor ${ }^{\mathrm{i}}$ and even specialized ones, such as GayCities,' they are labeled as 'gay' and 'male-dominated.

In contrast, Villa Mix (Figure 6(b)), and Blitz Haus are significantly more popular among female users. The manager of Villa Mix confirmed to us via email that they receive more visits of women than men. This might be explained by the fact that this nightclub frequently holds musical events with Sertanejo artists, a Brazilian music style that tendd to be popular among Brazilian women. It is important to mention that all venues studied in this section were contacted to confirm our results, and all the replies were mentioned in the text. For the case of Blitz Haus a fact that could help to explain the result is that according to their website, ${ }^{\mathrm{k}}$ the nightclub has a retro decoration, and besides music offers a gastronomic place.

This suggests that our methodology can detect venues that do not follow the same gender preference pattern observed in other venues of the same subcategory in the studied city. This result could be useful, for example, to improve venue classification schemes in the city.

Cultural differences, including those related to gender, may exist among different countries $[1-3,42]$. Besides that, there is a recent evidence that preferences for venues expressed in check-ins capture cultural differences among users [7]. Thus, differences of gender preferences for venues expressed in check-ins might also reflect different cultural patterns. In this direction, our methodology might be a useful tool to capture this partic- 
ular aspect of a certain culture, helping to leverage new types of applications, as discussed in the next section.

\section{Applications}

Many applications could benefit from our methodology to study gender preferences for venues. Some of them are:

Insights for policy-makers: Policy-makers could use the knowledge about gender preferences for venues to identify existing problems, and obtain insight into possible solutions for them, such as effective policies for gender differences reduction in certain regions or venues of the city.

New recommendation systems: The knowledge about cultural gender preferences for venues in a given city, neighborhood, or category of venues could be exploited in the design of new location recommendation services that take into account these preferences. These services could help tourists and residents find places of interest (e.g., where to go out in an unknown environment).

Understanding Consumers: Business owners and marketers could use the valuable insights about cultural gender preferences of specific venues or categories of venues, to promote more efficient advertisement.

Next, we present more details of an application that demonstrate one possibility to explore gender preferences for venues.

\subsection{Areas with similar gender popularity}

We here illustrate one particular application that aims at identifying groups of similar urban areas according to the degree of gender difference observed in the preference for different (types of) places located in those areas, where gender difference is inferred from the cross-gender popularity differences. As argued above, such popularity differences might reflect different cultural patterns. Thus, by clustering regions based on them, we aim at identifying groups of regions that share similar cultural traits related to gender preference for venues. This effort is similar to a recent investigation on using check-ins to identify cultural boundaries based on eating and drinking patterns [7], although we here explore a different cultural dimension.

Our goal in this section is to further investigate the extent to which our cross-gender popularity differences provide useful information about gender preference for venues in a given region of the real world. For that, the application we envision works as follows. We estimate the variability $w$ of the cross-gender popularity differences measured for all venues (in all subcategories) located in the region under study. A large $w$ across the venues is taken as a sign of large variability in the cross-gender popularity differences. ${ }^{1}$

To estimate $w$ we consider the Gini coefficient $(g)$, which was proposed to describe the income inequality in a population, but it can be used in the study of inequalities in several domains [45]. A Gini coefficient of zero expresses perfect equality, where all popularity differences values are the same. A Gini coefficient of one expresses maximal inequality among popularity differences values.

Mathematically, $g$ is defined based on the Lorenz curve, which plots, in our context, the proportion of popularity differences ( $y$ axis) that is cumulatively expressed by the $x \%$ of subcategories with smaller popularity differences, as shown by Figure 7 . The line at 45 degrees thus represents perfect equality of popularity differences. The Gini coefficient 
can then be thought of as the ratio of the area that lies between the line of equality and the Lorenz curve over the total area under the line of equality. Based on Figure 7, $g=A /(A+B)$.

To compute $g$ from an empirical Lorenz curve, one generated by discrete data points (our case), we can use the formula:

$$
g=\frac{n+1}{n}-\frac{2 \sum_{1}^{n}(n+1-i) x_{i}}{n \sum_{1}^{n} x_{i}}
$$

where the $x_{i}$ are the popularity differences ordered from least to greatest and $n$ is the number of popularity differences calculated. More details of the Gini Coefficient can be found in [45].

Given a set of regions $R$, we use the Gini metric to estimate the variability of the crossgender popularity differences for individual venues of each subcategory analyzed in each region $r \in R$. We then represent each region $r$ by a cultural gender preference vector, $G_{r}=\left\{g^{S_{1}}, g^{S_{2}}, \ldots, g^{S_{n}}\right\}$, where $g^{S_{i}}$ is the Gini coefficient computed for subcategory $S_{i}$, and $n$ is the total number of subcategories analyzed in all regions ( $n=299$, all subcategories considered). We assume $g^{S_{i}}=0$ if subcategory $S_{i}$ was not analyzed in region $r$ due to the lack of enough data. Finally, we use the $k$-means algorithm (with cosine distance) to cluster the regions in the space defined by $G_{r}$. The used data and code are available as a supplementary material of this study.

We tested this idea by clustering the 15 countries analyzed. First, we used $k=4$, as the countries are located in 4 distinct geographic regions of the world. Table 2 shows the iden-

Figure 7 Graphical representation of the Gini coefficient.

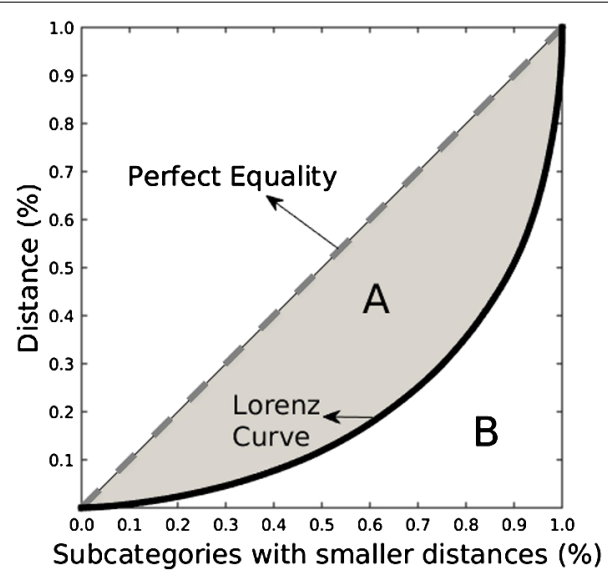

Table 2 Clustering of countries

\begin{tabular}{|c|c|c|c|}
\hline \multirow[t]{2}{*}{ Cluster } & $k=4$ & \multirow[t]{2}{*}{ Cluster } & \multirow{2}{*}{$\begin{array}{l}k=10 \\
\text { Countries }\end{array}$} \\
\hline & Countries & & \\
\hline \multirow[t]{2}{*}{1} & Saudi Arabia, United Arab Emirates, Kuwait & 1 & Saudi Arabia, Kuwait \\
\hline & & 2 & United Arab Emirates \\
\hline \multirow[t]{2}{*}{2} & Brazil, Mexico, United States, Japan, Malaysia, Thailand, Turkey & 3 & Turkey \\
\hline & & 4 & Brazil, Mexico \\
\hline \multirow[t]{2}{*}{3} & France, South Korea, United Kingdom & 5 & South Korea \\
\hline & & 6 & Malaysia, Thailand \\
\hline \multirow[t]{4}{*}{4} & Germany, Spain & 7 & Germany, Spain \\
\hline & & 8 & France \\
\hline & & 9 & United Kingdom \\
\hline & & 10 & Japan, United States \\
\hline
\end{tabular}


tified clusters. Some groupings are expected according to common sense. For example, all the Arab countries were grouped together, possibly because they share many cultural similarities regarding female habits. Yet, the table also reveals possibly unexpected results, such as the greater similarity of South Korea with European countries. Similarly, Thailand, Malaysia, and Turkey are grouped together with Brazil, Mexico, Japan, and United States. Despite the geographic (and perhaps also cultural), distance between some of the countries, they share similar patterns in cross-gender popularity differences, which might be a reflection of similar social conditions. In order to further investigate these results, we identified $k=10$ clusters, results also shown in Table 2. In this new grouping, UK, France, South Korea and Turkey represent a cluster by themselves, and Thailand and Malaysia is now a cluster, leaving Brazil and Mexico as another cluster. This result reinforces the suggestion that our data might indeed represent characteristics of the cultural behavior of the inhabitants of those places.

One could think that the result is correlated with the number of data available in the region of study, since some of the $k=4$ clusters, such as the one containing Germany, Spain, and France, have a small amount of data. However, if this was the case, South Korea and the United Arab Emirates would also be in the same cluster because they also have a small number of data. In order to further investigate this possible problem, we selected 19 popular cities according to the number of check-ins, representing distinct regions of the world: New York, Chicago, San Francisco (USA), Sao Paulo, Rio de Janeiro, Belo Horizonte (Brazil), Kuala Lumpur, Johor Bahru (Malaysia), Tokyo, Osaka (Japan), Paris (France), London (UK), Istanbul, Ankara, Izmir (Turkey), Riyadh, Jeddah (Saudi Arabia), Mexico City (Mexico), and Bangkok (Thailand).

Table 3(left) shows the results of clustering these cities using $k=10$, the same number of distinct countries where these cities are located. As we can see, most of the cities from the same country were clustered together. One exception, in this sense, was Istanbul grouped with San Francisco. Perhaps, the behavior of users of those cities is in fact more similar to each other than the other cities studied of the same country. Istanbul, due to the penalty mentioned in Section 4.2, presented a distinct pattern related to soccer places compared to other cities in the same country. The city is also concerned in promoting gender equality and the empowerment of women [46], and, maybe, some of the actions in this direction might have an effect, changing the behavior of inhabitants to be more similar to citizens of San Francisco. Besides that, today, Istanbul has the best record in regards to gender equality among 81 Turkish provinces [47]. Another exception was Kuala Lumpur grouped

Table 3 Clustering of cities

\begin{tabular}{llll}
\hline Cluster & $\boldsymbol{k}=\mathbf{1 0}$ & Cluster & $\boldsymbol{k}=\mathbf{2}$ \\
\cline { 2 - 2 } & Cities & 1 & Cities \\
\hline 1 & New York, Chicago & & New York, Chicago, San Francisco, Paris, \\
2 & Sao Paulo, Rio de Janeiro, Belo Horizonte & & Sao Paulo, Rio de Janeiro, Belo Horizonte, \\
3 & Johor Bahru, Riyadh, Jeddah & & Tokyo, Osaka, London, Mexico City \\
4 & Tokyo, Osaka & 2 & Kuala Lumpur, Johor Bahru, Istanbul, \\
5 & Kuala Lumpur, Bangkok & & Ankara, Izmir, Riyadh, Jeddah, Bangkok \\
6 & Istanbul, San Francisco & & \\
7 & Ankara, Izmir & & \\
8 & London & & \\
9 & Mexico City & & \\
10 & Paris & & \\
\hline
\end{tabular}


with Bangkok instead of Johor Bahru, which was grouped with Riyadh, Jeddah. The fact that Kuala Lumpur and Bangkok are bigger and more cosmopolitan cities might help to explain this clustering.

Note that by forcing the grouping into only 2 clusters (Table 3 - right), our strategy clearly distinguishes cities where most inhabitants have an Islamic tradition (cluster 2), which tends to shape a common cultural gender behavior, from the others. Our results suggest that the degree of gender preferences for venues might capture important aspects of gender inequality. Countries being in the same cluster were classified by sociologists with a similar gender inequality in the Gender Inequality Index (GII). We further investigate this question in the next section.

\section{Comparison with official indices}

Gender inequality can be defined as allowing people different opportunities due to perceived differences based solely on issues of gender [48]. This is a broad and complex definition and some initiatives attempt to measure it across different countries, such as the Gender Inequality Index (GII). GII is an index for measurement of gender inequality developed by the United Nations Development Programme (UNDP), being perhaps the most important study in this area. The index was introduced in the 2010 Human Development Report and we use in this study the 2014 index. GII is a value ranging from 0 (no perceivable inequality) to 1 (extreme inequality) reflecting the inequality between men and women in a given country. It is currently calculated for over 150 countries, which are ranked by the computed values. More details on calculation of GII can be found in [6].

We hypothesize that gender preferences for venues expressed in our data might reflect less contact between different genders (recall that we discarded categories that have many subcategories with expected biases towards a particular gender, e.g., Men's Store). This could affect networking opportunities and keep the 'glass ceilings' in society impermeable, aspects captured by studies of gender inequality such as GII. In this section, we investigate to which extent gender preferences for venues are related to gender inequality. To do that, we compare the results of our methodology with GII using the cultural gender preference vector, $G_{r}$, for a country $r$ considered in this study. For that, we rank for a given country $r$ all other countries according to a certain distance towards $r$. In the case of GII values we use Euclidean distance and for our vector, we use cosine distance. For example, assuming that $r=$ Brazil, we compute the Euclidean distance from GII value for Brazil to all other GII values for the other countries. After that, we compute the cosine distance from the vector representing Brazilians' preferences $\left(G_{\text {brazil }}\right)$ to all other preference vectors for other countries. Then, we compute a Spearman's rank correlation coefficient $\rho$ [49] between these two ranks, for each country (see Appendix 8 for more details). The idea is to verify if the most similar (and distinct) countries to a particular country in GII, for example, Brazil, are ranked similarly when we use the dimensions computed by our approach.

Furthermore, in order to verify if the observed relations are more pronounced for gender issues captured by GII, we also make the same comparison explained above using Human Development Index (HDI) and random data, replacing GII in the comparison. HDI is a composite statistic of life expectancy, education, and per capita income indicators. More details about how it is calculated can be found in [6]. In this study, we used HDI from 2014, the same year of our data collection. Since GII includes different dimensions than HDI, it cannot be interpreted as a loss or gain in HDI itself, i.e, it is unrelated to gender. 
Table 4 The correlation coefficient $\rho$ (and its $p$-value) between the rank of similarity generated from GIl and HDI with our approach. Significant and positive correlations are rendered in bold

\begin{tabular}{|c|c|c|c|c|c|}
\hline \multirow[t]{2}{*}{ Country } & \multicolumn{2}{|l|}{ GII } & \multicolumn{2}{|l|}{ HDI } & \multirow{2}{*}{$\begin{array}{l}\text { Random } \\
\text { Confidence interval (99\%) of } \rho\end{array}$} \\
\hline & $\rho$ & $p$-value & $\rho$ & $p$-value & \\
\hline Brazil & 0.665 & 0.011 & 0.573 & 0.035 & $(-0.051,0.071)$ \\
\hline France & 0.551 & 0.043 & 0.520 & 0.059 & $(-0.047,0.103)$ \\
\hline Germany & 0.134 & 0.648 & 0.024 & 0.939 & $(-0.074,0.058)$ \\
\hline Japan & -0.569 & 0.036 & -0.564 & 0.038 & $(-0.037,0.093)$ \\
\hline Kuwait & 0.709 & 0.006 & 0.564 & 0.038 & $(-0.098,0.044)$ \\
\hline Malaysia & -0.345 & 0.227 & 0.670 & 0.010 & $(-0.070,0.071)$ \\
\hline Mexico & 0.589 & 0.026 & 0.446 & 0.111 & $(-0.090,0.049)$ \\
\hline Saudi Arabia & 0.558 & 0.037 & -0.277 & 0.337 & $(-0.152,-0.002)$ \\
\hline South Korea & 0.653 & 0.011 & 0.556 & 0.050 & $(-0.014,0.117)$ \\
\hline Spain & 0.547 & 0.045 & 0.363 & 0.202 & $(-0.067,0.072)$ \\
\hline Thailand & 0.675 & 0.008 & 0.758 & 0.002 & $(-0.081,0.057)$ \\
\hline Turkey & 0.753 & 0.002 & 0.661 & 0.012 & $(-0.079,0.043)$ \\
\hline UAE & -0.116 & 0.693 & 0.314 & 0.273 & $(-0.111,0.034)$ \\
\hline United Kingdom & 0.107 & 0.715 & 0.187 & 0.522 & $(-0.017,0.126)$ \\
\hline United States & 0.279 & 0.333 & -0.516 & 0.061 & $(-0.108,0.033)$ \\
\hline
\end{tabular}

To generate random data we randomly ordered the considered countries. Let $V$ represent a particular rank, in our case we use the values for GII in Table 6 from Appendix 8, where each line represents a country. We use a function $f$ to perform a random permutation in that vector: $V^{\prime}=f(V)$, where $V^{\prime}$ represent a particular permutation of $V$. We created 100 random ranks: $\mathcal{R}=\left\{V_{1}^{\prime}, V_{2}^{\prime}, \ldots, V_{n}^{\prime}\right\}$, where $n=100$. We compared every $V_{i}^{\prime} \in \mathcal{R}$ with our data, resulting in $100 \rho$ correlation values.

The results are shown in Table 4. The first column lists the countries considered, while the second to fifth show the correlation performed $\rho$ and it's respective $p$-value, for GII and HDI. We highlight in bold all the coefficients that are positive and statistically significant, i.e., with a $p$-value $<0.05$. For example, the first line for GII presents the result of the Spearman correlation from the two ranks produced in the example aforementioned for Brazil. In other words, the rank produced of distances from Brazil to the other studied countries for GII values and our preference vectors has a Spearman correlation value of 0.665 , and this value is significant. The sixth column represent a $99 \%$ confidence interval of the mean $\rho$ relative to $\mathcal{R}$.

Note in Table 4 that a majority of countries show a positive and significant correlation $\rho$ between our gender preference measure with the GII ( 9 out of 15 countries). In contrast, fewer countries ( 5 out of 15 ) have a positive and significant correlation with the HDI. In addition, most of the positive correlation values are higher for the GII case. Random rankings show no correlation (i.e., $\rho$ close to 0 ), as expected. The results suggest the outcomes observed are not explained by a general cultural similarity between countries. Besides, they indicate that cross-gender popularity differences, relying solely on check-in data, might capture important aspects of gender inequality that emerge in sophisticated studies, such as GII. It is important to mention that there are cases where the proposed method does not seem to be related to the GII. For instance, we can find a significant negative correlation for the case of Japan, which we also observed for the correlation with the HDI. Despite of that, the results suggest that our proposed methodology could be exploited to complement existing methodologies to study gender inequalities, for instance, as an additional dimension. However, further research is needed. 


\section{Limitations}

There are several possible reasons for results observed in the comparison (Section 6) and also in the clustering results (Section 5.1). Some countries in our dataset have a small number of users (and check-ins), possibly reflecting a lower adoption of Foursquare among those countries' inhabitants. This is a limitation of our dataset, which covers only seven days. A dataset spanning a longer period would most certainly capture a larger fraction of the population of those countries, although the adoption rate imposes inherent constraints. Besides that, there might be more accurate methods than the Gini coefficient to generate the cultural gender preference vector, other metrics could also be tested aiming to improve the comparison results. Yet, our methodology also has limitations. Take, for instance, Saudi Arabia, where the same place may have exclusive sectors for men and women, such as restaurants with segregated service and eating zones, and shopping malls with dedicated floors for women (as in the Kingdom Centre ${ }^{\mathrm{m}}$ ). The gender segregation in those places is very high. Yet, our approach is not able to capture the correct level of segregation since those gender-specific sectors and zones are not distinguished as different venues on Foursquare.

Besides that, our methodology assumes that the gender information given by users on their profile page are correct. This might not be a significant problem since there is evidence that users provide correct gender information in their online profiles. Burger et al. [32] studied user gender on Twitter considering gender information shared by users in external blog accounts associated with their Twitter account. This association enabled an experiment verifying that cues in Twitter profile descriptions, e.g. 'mother of 3 children', tend to be consistent with gender information in the blog. This may indicate that people who misrepresent their gender are consistent across different aspects of their online presence. Linked to that, our proposed methodology also does not tackle the case where users do not fit in either male or female gender, as shown by [36]. Our methodology also does not treat pollution, e.g. fake accounts. In this particular case, techniques to increase data quality could improve the results [50-52].

\section{Conclusions and future work}

We have proposed a methodology to identify gender differences in preferences for specific venues in urban regions by analyzing user check-in data on Foursquare. We illustrated the use of our methodology by applying it to identify statistically significant cross-gender differences in preferences for venues, at both country and city levels. Some of these significant differences reflect well-known cultural patterns, while others could be explained by particular aspects of the venues identified after manual research.

We also gathered evidence that our methodology offers useful information about gender preference for venues in a given region in the real world. This result suggests that, despite limitations and biases that might exist in our data, our methodology could be a useful tool to support faster and cheaper large-scale studies on gender preferences for venues.

By exploiting our cross-gender preferences for venue differences, business owners could gain valuable insights about their customers; venue recommendations could become more culturally-aware, as men and women may have different preferences in regions with distinct cultures; and data-intensive sociological studies about gender preferences for venues could be done in less time, with larger sample sizes, and on regions with arbitrary spatial granularities. 
As future work, we intend to validate our methodology with other LBSN datasets and other data about gender preferences for venues collected in a traditional (offline) fashion. Besides that, we envision to investigate how the proposed methodology could be exploited to complement existing methodologies to study gender inequalities. We also plan to investigate other applications that can benefit from our results, and expand our methodology to add a temporal dimension, thus capturing temporal variations in cross-gender preferences for venues that might exist.

\section{Appendix: details about the comparison with official indices}

This appendix shows extra information about the comparison with official indices performed in Section 6. The data for the Gender Inequality Index and Human Development Index were obtained on the UNDP website (hdr.undp.org). All data refer to the year of 2014. For reference, data for each country studied in this work are presented in Table 6.

To perform the comparison considered in Section 6 we have to rank for a given country $r$ all other countries according to a certain distance towards $r$. To illustrate this process, consider $r=$ Brazil. The first step is to calculate the Euclidean distance vector $D 1_{r}$ from Brazil to all other countries according to GII. ${ }^{\mathrm{n}}$ In other words, we compute the pairwise $\mathrm{Eu}$ clidean distance between pairs of country data. According to our example, Brazil has GII value of 0.457 (Table 6), and we have to compute the distance for all other countries. The result for this example is $D 1_{\text {Brazil }}=\{0,0.369,0.416,0.324,0.070,0.248,0.084,0.173,0.332$, $0.362,0.077,0.098,0.225,0.280,0.177\}$.

After that, we compute the cosine distance ${ }^{\circ} D 2_{r}$ from the vector representing the preferences of Brazilians ( $G_{\text {brazil }}$ ) to all other preference vectors for other countries. In our example it is $D 2_{r}=\{0,0.755,0.757,0.415,0.556,0.328,0.249,0.564,0.796,0.73,0.324,0.379$, $0.795,0.601,0.379\}$. For reference, Table 5 shows the cosine distance from a preference vector representing a certain country to all other preference vectors representing the other countries. Then, we compute a Spearman's rank correlation coefficient $\rho$ [49] between these two ranks, for each country. But, before that, we disregard the distance from $r$ itself, which in our example is located in the first position of the distance vectors. The correlation coefficient $\rho$ for this example, as shown in Table 4, is 0.66 (with a $p$-value of 0.01 ). The code and data used to perform this analysis are provided as a supplementary material.

Table 5 Cosine distance from a preference vector for a specific country to all preference vectors for all other countries

\begin{tabular}{llllllllllllllll}
\hline & BR & FR & GE & JA & KU & MA & ME & SA & SK & SP & TH & TU & UAE & UK & USA \\
\hline Brazil (BR) & 0 & 0.755 & 0.757 & 0.415 & 0.556 & 0.328 & 0.249 & 0.564 & 0.796 & 0.73 & 0.324 & 0.379 & 0.795 & 0.601 & 0.379 \\
France (FR) & 0.755 & 0 & 0.886 & 0.678 & 0.891 & 0.806 & 0.781 & 1 & 0.656 & 0.497 & 0.775 & 0.798 & 0.747 & 0.684 & 0.765 \\
Germany (GE) & 0.757 & 0.886 & 0 & 0.7 & 0.873 & 0.885 & 0.796 & 0.894 & 0.56 & 0.381 & 0.794 & 0.831 & 0.777 & 0.837 & 0.803 \\
Japan (JA) & 0.415 & 0.678 & 0.7 & 0 & 0.655 & 0.457 & 0.43 & 0.677 & 0.689 & 0.689 & 0.445 & 0.552 & 0.779 & 0.585 & 0.339 \\
Kuwait (KU) & 0.556 & 0.891 & 0.873 & 0.655 & 0 & 0.536 & 0.611 & 0.359 & 0.825 & 0.939 & 0.6 & 0.572 & 0.6 & 0.88 & 0.7 \\
Malaysia (MA) & 0.328 & 0.806 & 0.885 & 0.457 & 0.536 & 0 & 0.341 & 0.488 & 0.893 & 0.863 & 0.362 & 0.407 & 0.757 & 0.782 & 0.467 \\
Mexico (ME) & 0.249 & 0.781 & 0.796 & 0.43 & 0.611 & 0.341 & 0 & 0.581 & 0.778 & 0.749 & 0.429 & 0.394 & 0.824 & 0.604 & 0.273 \\
Saudi Arabia (SA) & 0.564 & 1 & 0.894 & 0.677 & 0.359 & 0.488 & 0.581 & 0 & 0.936 & 1 & 0.506 & 0.509 & 0.712 & 0.934 & 0.653 \\
South Korea (SK) & 0.796 & 0.656 & 0.56 & 0.689 & 0.825 & 0.893 & 0.778 & 0.936 & 0 & 0.497 & 0.786 & 0.706 & 0.639 & 0.52 & 0.71 \\
Spain (SP) & 0.73 & 0.497 & 0.381 & 0.689 & 0.939 & 0.863 & 0.749 & 1 & 0.497 & 0 & 0.714 & 0.825 & 0.858 & 0.737 & 0.717 \\
Thailand (TH) & 0.324 & 0.775 & 0.794 & 0.445 & 0.6 & 0.362 & 0.429 & 0.506 & 0.786 & 0.714 & 0 & 0.421 & 0.72 & 0.731 & 0.52 \\
Turkey (TU) & 0.379 & 0.798 & 0.831 & 0.552 & 0.572 & 0.407 & 0.394 & 0.509 & 0.706 & 0.825 & 0.421 & 0 & 0.766 & 0.577 & 0.492 \\
UAE & 0.795 & 0.747 & 0.777 & 0.779 & 0.6 & 0.757 & 0.824 & 0.712 & 0.639 & 0.858 & 0.72 & 0.766 & 0 & 0.877 & 0.811 \\
UK & 0.601 & 0.684 & 0.837 & 0.585 & 0.88 & 0.782 & 0.604 & 0.934 & 0.52 & 0.737 & 0.731 & 0.577 & 0.877 & 0 & 0.558 \\
USA & 0.379 & 0.765 & 0.803 & 0.339 & 0.7 & 0.467 & 0.273 & 0.653 & 0.71 & 0.717 & 0.52 & 0.492 & 0.811 & 0.558 & 0 \\
\hline
\end{tabular}




\section{Table 6 Considered data for Gender Inequality Index and Human Development Index}

\begin{tabular}{lll}
\hline Country & Gll value & HDI value \\
\hline Brazil & 0.457 & 0.755 \\
France & 0.088 & 0.888 \\
Germany & 0.041 & 0.916 \\
Japan & 0.133 & 0.891 \\
Kuwait & 0.387 & 0.816 \\
Malaysia & 0.209 & 0.779 \\
Mexico & 0.373 & 0.756 \\
Saudi Arabia & 0.284 & 0.837 \\
South Korea & 0.125 & 0.898 \\
Spain & 0.095 & 0.876 \\
Thailand & 0.38 & 0.726 \\
Turkey & 0.359 & 0.761 \\
United Arab Emirates & 0.232 & 0.835 \\
United Kingdom & 0.177 & 0.907 \\
United States & 0.28 & 0.915 \\
\hline
\end{tabular}

\section{Competing interests}

The authors declare that they have no competing interests.

\section{Authors' contributions}

WM, THS, JMA, AAFL conceived, designed, and coordinated the study; WM and THS carried out data processing; WM and THS performed statistical analysis and visualization of results. All the authors interpreted the results, wrote the manuscript and gave the final approval for publication.

\section{Author details}

${ }^{1}$ Department of Computer Science, Hasso-Plattner-Institut, Potsdam, Brandenburg, Germany. ${ }^{2}$ Department of Computer Science, Universidade Federal de Minas Gerais, Belo Horizonte, MG, Brazil. ${ }^{3}$ Present address: Department of Informatics, Universidade Tecnológica Federal do Paraná, Curitiba, PR, Brazil.

\section{Acknowledgements}

This work was partially supported by the FAPEMIG-PRONEX-MASWeb project - Models, Algorithms and Systems for the Web, process number APQ-01400-14, as well as by the National Institute of Science and Technology for the Web (INWEB), Capes, CNPq, FAPEMIG, and Fundação Araucária.

\section{Endnotes}

a http://www.foursquare.com.

b We did experiment with other approaches to computing the popularity difference, such as the difference between the coordinates but the results are similar.

c In this figure and also in Figures 2 and 4 ' $*$ ' means that the difference observed is statistically significant.

d https://www.opendemocracy.net/can-europe-make-it/aslan-amani/football-in-turkey-force-for-liberalisation-andmodernity.

e http://www.dailymail.co.uk/sport/football/article-2614502/Turkish-delight-Fenerbahce-wrap-19th-league-title-win50-000-women-children.html.

f http://www.theguardian.com/music/2011/dec/15/cowell-pop-k-pop.

g http://www.worldpolicy.org/blog/2011/10/18/higher-education-path-progress-saudi-women.

h http://www.yelp.com.

i http://www.tripadvisor.com

j http://www.gaycities.com

k http://blitzhaus.com.br.

I We note that the cross-gender popularity differences might be equally large in all venues, resulting in low variability. Our strategy does not catch those cases. However, this pattern is unlikely to happen in practice, and indeed we did not observe it in our dataset.

m http://kingdomcentre.com.sa/ladies.html.

$\mathrm{n}$ For simplicity we consider in this example only data for Gll, but the same procedure has to be performed when considering $\mathrm{HDI}$ or random data.

O One minus the cosine of the angle between the considered vectors.

\section{Publisher's Note}

Springer Nature remains neutral with regard to jurisdictional claims in published maps and institutional affiliations. 


\section{References}

1. Sen A (2001) The many faces of gender inequality. New republic, pp 35-39

2. Szymanowicz A, Furnham A (2011) Do intelligent women stay single? Cultural stereotypes concerning the intellectual abilities of men and women. Journal of Gender Studies 20(01):43-54

3. Harrison LE, Huntington SP (2000) Culture matters: how values shape human progress. Basic Books, New York

4. Hyde JS (2005) The gender similarities hypothesis. Am Psychol 60(6):581-592

5. Ridgeway $\mathrm{CL}$ (2011) Framed by gender: how gender inequality persists in the modern world. Oxford University Press, London

6. United Nations Development Programme: Human Development Reports (2015) http://hdr.undp.org/

7. Silva T, Vaz de Melo P, Almeida J, Musolesi M, Loureiro A (2014) You are what you eat (and drink): identifying cultural boundaries by analyzing food \& drink habits in Foursquare. In: Proc. of ICWSM'14. AAAI, Ann Arbor, USA

8. Cranshaw J, Schwartz R, Hong JI, Sadeh N (2012) The livehoods project: utilizing social media to understand the dynamics of a city. In: Proc. of ICWSM'12. AAAl, Dublin, Ireland

9. Baron NS, Campbell EM (2010) Talking takes too long: gender and cultural patterns in mobile telephony. In: Conf. of Assoc. of Internet Researchers, Göteborg, Sweden

10. Garcia D, Ingmar Weber VG (2014) Gender asymmetries in reality and fiction: the bechdel test of social media. In: Proc. of ICWSM'14. AAAl, Ann Arbor, USA

11. Žižek S (1989) The sublime object of ideology. Verso, London

12. Magno G, Weber I (2014) In: International gender differences and gaps in online social networks, Barcelona, Spain, pp 121-138

13. Wagner C, Garcia D, Jadidi M, Strohmaier M (2015) It's a man's wikipedia? assessing gender inequality in an online encyclopedia. arXiv preprint. arXiv:1501.06307

14. Volkovich Y, Laniado D, Kappler KE, Kaltenbrunner A (2014) Proc. of SocInfo'14 Springer, Barcelona, Spain, pp 139-150

15. Buchmann C, DiPrete TA, McDaniel A (2008) Gender inequalities in education. Annu Rev Sociol 34:319-337

16. van Hooff JH (2011) Rationalising inequality: heterosexual couples' explanations and justifications for the division of housework along traditionally gendered lines. J Gend Stud 20(01):19-30

17. Hargittai E, Hinnant A (2008) Digital inequality differences in young adults' use of the Internet. Commun Res 35(5):602-621

18. Kershaw D, Rowe M, Stacey P (2014) Towards tracking and analysing regional alcohol consumption patterns in the UK through the use of social media. In: Proc. of WebSci'14. ACM, Bloomington, USA, pp 220-228. doi:10.1145/2615569.2615678

19. Weber I, Garimella VRK, Borra E (2012) Mining web query logs to analyze political issues. In: Proc. of WebSci '12, Evanston, USA, pp 330-334. doi:10.1145/2380718.2380761

20. Zambaldi V, Pesce JP, Quercia D, Almeida V (2014) Lightweight contextual ranking of city pictures: urban sociology to the rescue. In: Proc. of ICWSM'14. AAAl, Ann Arbor, USA

21. Silva TH, Vaz de Melo POS, Almeida JM, Salles J, Loureiro AAF (2014) Revealing the city that we cannot see. ACM Trans Internet Technol 14(4):26:1-26:23. doi:10.1145/2677208

22. Georgiev P, Noulas A, Mascolo C (2014) The call of the crowd: event participation in location-based social services. In: Proc. of ICWSM'14. AAAI, Ann Arbor, USA

23. Sakaki T, Okazaki M, Matsuo Y (2010) Earthquake shakes Twitter users: real-time event detection by social sensors. In: Proc. of WWW'10. ACM, Raleigh, USA, pp 851-860

24. Alsaedi N, Burnap P, Rana OF (2014) A combined classification-clustering framework for identifying disruptive events. In: Proc. of SocialCom'14, Stanford, USA

25. Becker H, Naaman M, Gravano L (2011) Beyond trending topics: real-world event identification on Twitter. In: Proc. of ICWSM'11. AAAl, Barcelona, Spain

26. Pan B, Zheng Y, Wilkie D, Shahabi C (2013) Crowd sensing of traffic anomalies based on human mobility and social media. In: Proc. of SIGSPATIAL'13. ACM, Orlando, Florida, pp 344-353

27. Gomide J, Veloso A, Meira W Jr, Almeida V, Benevenuto F, Ferraz F, Teixeira M (2011) Dengue surveillance based on a computational model of spatio-temporal locality of Twitter. In: Proc. of WebSci'11. ACM, Evanston, USA

28. Garcia-Gavilanes R, Quercia D, Jaimes A (2013) Cultural dimensions in Twitter: time, individualism and power. In: Proc. of ICWSM'13. AAAl, Boston, USA

29. Hochman N, Schwartz R (2012) Visualizing instagram: tracing cultural visual rhythms. In: Proc. of work. on social media vis. AAAl, Dublin, Ireland, pp 6-9

30. Mocanu D, Baronchelli A, Perra N, Gonçalves B, Zhang Q, Vespignani A (2013) The Twitter of babel: mapping world languages through microblogging platforms. PLoS ONE 8(4) e61981

31. Ciot M, Sonderegger M, Ruths D (2013) Gender inference of Twitter users in non-English contexts. In: Proc. of EMNLP'13, Seattle, USA, pp 1136-1145

32. Burger JD, Henderson J, Kim G, Zarrella G (2011) Discriminating gender on Twitter. In: Proc. of EMNLP'11, Edinburgh, United Kingdom, pp 1301-1309

33. Liu W, Ruths D (2013) What's in a name? Using first names as features for gender inference in Twitter. In: Symp. on analyzing microtext, Stanford, USA

34. Ottoni R, Pesce JP, Las Casas DB, Franciscani G Jr, Meira W Jr, Kumaraguru P, Almeida V (2013) Ladies first: analyzing gender roles and behaviors in pinterest. In: Proc. of ICWSM'13. AAAI, Boston, USA

35. Lou JK, Park K, Cha M, Park J, Lei CL, Chen KT (2013) Gender swapping and user behaviors in online social games. In: Proc. of WWW'13. ACM, Rio de Janeiro, Brazil, pp 827-836

36. de Las Casas DC, Magno G, Cunha E, Gonçalves MA, Cambraia C, Almeida V (2014) Noticing the other gender on Google+. In: Proc. of WebSci '14. ACM, Bloomington, USA, pp 156-160

37. Cunha E, Magno G, Almeida V, Gonçalves MA, Benevenuto F (2012) A gender based study of tagging behavior in Twitter. In: Proc. of HT'12. ACM, Milwaukee, USA, pp 323-324

38. Graells-Garrido E, Lalmas M, Menczer F (2015) First women, second sex: gender bias in Wikipedia. In: Proc. of HT '15. ACM, Guzelyurt, Northern Cyprus, pp 165-174. doi:10.1145/2700171.2791036

39. Hofstede GH (2001) Culture's consequences: Comparing values, behaviors, institutions and organizations across nations. Sage 
40. Jackson S (2011) Research methods and statistics: A critical thinking approach. Cengage Learning

41. Vianello M, Siemienska R, Damian N, Lupri E, Coppi R, D’Arcangelo E, Bolasco S (1990) Gender inequality: a comparative study of discrimination and participation. Sage Publications, Thousand Oaks

42. Inglehart R, Welzel C (2010) Changing mass priorities: the link between modernization and democracy. Perspective Polit 8(02):551-567. doi:10.1017/s1537592710001258

43. Akyar A (2012) Sociability in Starbucks coffee houses of Istanbul the contemporary public space and its uses. Master's thesis, Central European University, Budapest

44. Fundação Bienal de São Paulo: Relatório de Gestão 2013-2014 (2015). https://goo.gl/yM6qOd

45. Ceriani L, Verme P (2012) The origins of the Gini index: extracts from variabilità e mutabilità (1912) by corrado Gini. J Econ Inequal 10(3):421-443. doi:10.1007/s10888-011-9188-x

46. Women UN (2016) Gender equality and the Istanbul programme of action. https://goo.gl/HhFlsj

47. Boyacioğlu H (2016) Istanbul again top Turkish city for gender equality. Hurriyet Daily News. https://goo.gl/8oWqoU

48. Parziale A (2008) Gender inequality and discrimination. Encyclopedia of business ethics and society, 978-981

49. Jain R (2008) The art of computer systems performance analysis. Wiley, India

50. Ghosh S, Viswanath B, Kooti F, Sharma NK, Korlam G, Benevenuto F, Ganguly N, Gummadi KP (2012) Understanding and combating link farming in the Twitter social network. In: Proc. of WWW '12. ACM, Lyon, France, pp 61-70. doi:10.1145/2187836.2187846

51. Gupta A, Lamba H, Kumaraguru P, Joshi A (2013) Faking sandy: characterizing and identifying fake images on Twitter during hurricane sandy. In: Proc. of WWW'13 companion. ACM, Rio de Janeiro, Brazil, pp 729-736. doi:10.1145/2487788.2488033

52. Yang Z, Wilson C, Wang X, Gao T, Zhao BY, Dai Y (2014) Uncovering social network Sybils in the wild. ACM Trans Knowl Discov Data 8(1):2:1-2:29. doi:10.1145/2556609

\section{Submit your manuscript to a SpringerOpen ${ }^{\odot}$ journal and benefit from:}

- Convenient online submission

- Rigorous peer review

- Immediate publication on acceptance

- Open access: articles freely available online

- High visibility within the field

- Retaining the copyright to your article 